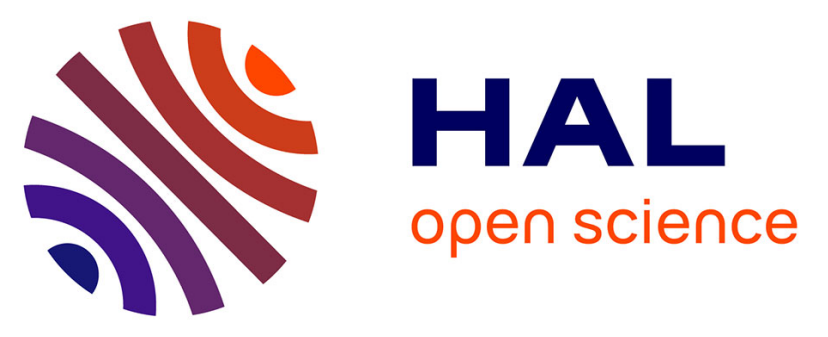

\title{
Echo-Power Estimation from Log-Compressed Video Data in Dynamic Contrast-Enhanced Ultrasound Imaging
}

\author{
Thomas Payen, Alain Coron, Michele Lamuraglia, Delphine Le \\ Guillou-Buffello, Emmanuel Gaud, Marcel Arditi, Olivier Lucidarme, S Lori \\ Bridal
}

\section{To cite this version:}

Thomas Payen, Alain Coron, Michele Lamuraglia, Delphine Le Guillou-Buffello, Emmanuel Gaud, et al.. Echo-Power Estimation from Log-Compressed Video Data in Dynamic ContrastEnhanced Ultrasound Imaging. Ultrasound in Medicine \& Biology, 2013, 39 (10), pp.1826-1837. 10.1016/j.ultrasmedbio.2013.03.022 . hal-01161941

\section{HAL Id: hal-01161941 https://hal.sorbonne-universite.fr/hal-01161941}

Submitted on 9 Jun 2015

HAL is a multi-disciplinary open access archive for the deposit and dissemination of scientific research documents, whether they are published or not. The documents may come from teaching and research institutions in France or abroad, or from public or private research centers.
L'archive ouverte pluridisciplinaire HAL, est destinée au dépôt et à la diffusion de documents scientifiques de niveau recherche, publiés ou non, émanant des établissements d'enseignement et de recherche français ou étrangers, des laboratoires publics ou privés. 


\section{Echo-power estimation from log-compressed video-data in dynamic contrast-enhanced ultrasound imaging}

Thomas Payen $^{1,2}$, Alain Coron ${ }^{1,2}$, Michele Lamuraglia ${ }^{1,2,3}$, Delphine Le Guillou-Buffello ${ }^{1,2}$, Emmanuel Gaud ${ }^{4}$, Marcel Arditi ${ }^{4}$, Olivier Lucidarme ${ }^{5}$, S. Lori Bridal ${ }^{1,2}$

${ }^{1}$ Université Pierre et Marie Curie, F-75005, Paris, France

${ }^{2}$ CNRS, UMR 7623, Laboratoire d'Imagerie Paramétrique, F-75006, Paris, France

${ }^{3}$ Medical Oncology Department, Georges Pompidou European Hospital, Paris, France

${ }^{4}$ Bracco Suisse SA, Geneva, Switzerland

${ }^{5}$ Department of Radiology, GH Pitié-Salpêtrière, Assistance Publique-Hôpitaux de Paris, Functional Imaging Laboratory, INSERM UPMC 678

Corresponding author:

Lori Bridal

Laboratoire d'Imagerie Paramétrique UMR 7623

15 rue de l'Ecole de Médecine

75006 Paris

France

Email: lori.bridal@upmc.fr

Tel: (+33) 144419605 


\section{Abstract}

Ultrasound (US) scanners typically apply lossy, nonlinear modifications to the US data for visualization purposes. The resulting images are then stored as compressed video data. Some system manufacturers provide dedicated software for quantification purposes, in order to eliminate such processing distortions, at least partially. This is currently the recommended approach for quantitatively assessing changes in contrast-agent concentration from clinical data. However, the machine-specific access to US data and the limited set of analysis functionalities offered by each dedicated-software package make it difficult to perform comparable analyses with different US systems. The objective of this work was to establish if linearization of compressed video images obtained with an arbitrary US system can provide an alternative to dedicated-software analysis of machine-specific files for the estimation of echo-power. For this purpose, a Toshiba Aplio 50 system was used, coupled with dedicated CHI-Q (Contrast Harmonic Imaging Quantification) software by Toshiba Medical Systems. Results were compared with two approaches that apply algorithms to estimate relative echo-power from compressed video images: commercially-available VueBox ${ }^{\mathrm{TM}}$ software by Bracco Suisse SA and inlaboratory software called PixPower. The echo-power estimated by $\mathrm{CHI}-\mathrm{Q}$ analysis demonstrated a strong linear relationship vs. agent concentration in vitro $\left(R^{2} \geq 0.9996\right)$ for dynamic range settings of $D R 60$ and $D R 80$, with slopes between 9.22 and $9.57 \mathrm{~dB} /$ decade $(p=0.05)$. These values approach the theoretically-predicted dependence of $10.0 \mathrm{~dB} /$ decade (equivalent to $3 \mathrm{~dB}$ for each concentration doubling). Echo-power estimations obtained from compressed video images with VueBox ${ }^{\mathrm{TM}}$ and PixPower also showed strong linear proportionality with concentration $\left(R^{2} \geq 0.9996\right)$, with slopes between 9.30 and $9.68 \mathrm{~dB} / \mathrm{decade}(\mathrm{p}=0.05)$. On an independent in vivo data set $(\mathrm{N}=24)$, the difference in echo-power estimation between $\mathrm{CHI}-\mathrm{Q}$ and each of the other two approaches was calculated after excluding regions which contain pixels values affected by saturated or thresholded pixel values. The mean difference in estimates (expressed in decibels) was $-0.25 \mathrm{~dB}$ between VueBox ${ }^{\mathrm{TM}}$ and $\mathrm{CHI}-\mathrm{Q}$ (95\% confidence interval from -0.75 to $0.26 \mathrm{~dB}$ ) and $-0.17 \mathrm{~dB}$ between PixPower and $\mathrm{CHI}-\mathrm{Q}$ ( $95 \%$ confidence interval from -0.67 to $0.13 \mathrm{~dB}$ ). To achieve linearization of data, one of the approaches $\left(\right.$ VueBox ${ }^{\mathrm{TM}}$ ) requires calibration files provided by the software manufacturer for each machine type and setting. The other (PixPower) requires empirical correction of the imaging dynamic range (DR) based on ground truth data. These requirements could potentially be removed if US systems were willing to make relevant information on the applied processing publicly available. Reliable echo-power estimation from linearized data would facilitate inclusion of different US systems in multicentric studies and more widespread implementation of emerging techniques for quantitative analysis of contrast-ultrasound.

Keywords: contrast-enhanced ultrasound, echo-power, linearization, log-compression, microbubbles, perfusion quantification, video-compression 


\section{Introduction}

Quantifying organ perfusion is essential in diagnosing and treating diseases such as cancer (Jain et al.1997) and cardiovascular dysfunctions (Blankstein et al.2009;Chung et al.2010;Folkman1995). Non invasive imaging has a central role to play in the clinical assessment and repeated follow-up measurements of perfusion in tumors or organs. Among the many imaging modalities available, ultrasound (US) is of particular interest because it is a safe, bed-side and well-accepted technique that can be repeated often. Recent studies have shown that Dynamic Contrast-Enhanced Ultrasound (DCE-US) offers the possibility to obtain perfusion information by assessing contrast agent uptake (Dietrich et al.2012;Gauthier et al.2012a;Guibal et al.2010; Lamuraglia et al.2010; Leen et al.2012; Quaia2007). Over a large range of concentrations, the echopower resulting from the contrast microbubbles' acoustic response is proportional to their concentration in the region of interest (ROI) (de Jong and Hoff1993;Lampaskis and Averkiou2010;Moran et al.2002). Detection of microbubble tracers in the blood and appropriate perfusion models can, thus, provide a valuable assessment of microvascular flow and relative blood volume (Arditi et al.2006;Hudson et al.2011;Lucidarme et al.2003;Wei et al.1998). These flow parameters cannot be reliably estimated, however, if robust measurements of the echopower are not obtained from the DCE-US sequences.

The lack of accessibility to machine-dependent echo signals can make it difficult to compare analyses of data from different US systems, between departments within a hospital or in multicentric studies. Raw Radio Frequency (RF) signal and Quadrature (IQ) signal (Powers et al.1980) analyses are the gold standards for echopower estimation. However, RF and IQ signals are generally not made available on machines for clinical use, largely due to the large storage space required to handle this type of data. US systems usually perform a variety of processing operations on the echo signal for visualization purposes as described in Figure 1. Demodulated echo signals are typically log-compressed to remap the dynamic range of meaningful ultrasound echoes at each depth ( $\sim 60-70 \mathrm{~dB})$ to the range of perception for the human eye of a monitor display ( $30 \mathrm{~dB})$ (Kremkau1998). Results are then provided as compressed video images in DICOM (Digital Imaging and Communications in Medicine) JPEG files (DICOM JPEG). DICOM JPEG files are generally considered unsuitable for echo-power estimation for two main reasons. First, straightforward echo-power estimation from DICOM JPEG files is hindered by a lack of publically available information on the process used to create the displayed color images.

In particular, information on the log-compression is not available (Gauthier et al.2011;Rognin et 
al.2008; Sanches and Marques2003). Second, in addition to the reversible logarithmic compression, DICOM JPEG data has undergone steps of thresholding and quantization during which information is lost.

Dedicated software produced by scanner manufacturers is able to access US data prior to final display processing and read proprietary information on processing stages for optimized linearization of ultrasonic data. Analysis with such software is currently the recommended method for assessing changes in contrast-agent concentration in a quantitative way (Gauthier et al.2011;Lassau et al.2007;Peronneau et al.2010). One example of such software is CHI-Q (Contrast Harmonic Imaging Quantification software, Toshiba Medical Systems, Tochigi, Japan) that is used to read files that we will refer to as DICOM Raw Data. Machine-specific software, however, is not available for every system. Lack of flexibility of these dedicated software in terms of region selection and analysis models further impede comparison of analysis with different US systems.

Software that applies echo-power estimation algorithms to DICOM JPEG data is available (Gauthier et al.2012b;Krix et al.2003;Lucidarme et al.2001;Tranquart et al.2012) or under development. This second analysis option can potentially provide more flexible integration of new sequence-analysis approaches and enable analysis of contrast data acquired from a large range of imaging systems. However, it has not yet been demonstrated that echo-power estimation from DICOM JPEG files with such algorithms can provide results which are equivalent to estimations based on DICOM Raw Data. The objective of this work was to determine whether linearization of compressed DICOM JPEG can provide estimates of the relative echo-power as good as those obtained using dedicated software to analyze DICOM Raw Data files. DCE-US image sets were obtained simultaneously in DICOM JPEG and DICOM Raw Data files using the Toshiba Aplio 50 US system. The techniques developed and the criteria applied to achieve linearization of DICOM JPEG data are described.

Three approaches for estimation of echo-power were compared: CHI-Q analyzing DICOM Raw Data, the VueBox $^{\text {TM }}$ software package (Bracco Suisse SA, Geneva, Switzerland) processing DICOM JPEG images and the PixPower software package (developed in-house at the UPMC and CNRS, UMR 7623) that also uses DICOM JPEG images.

First, in vitro experiments were conducted at two different dynamic ranges with the three approaches to compare the proportionality between echo-power estimates and the concentration of contrast agent microbubbles in suspension. Agreement between the echo-power estimated by the three approaches was then evaluated using DCE-US sequences acquired in an in vivo tumor model. Care was taken in both experiments to compare echo-power estimates throughout the full dynamic range. The results validate the two techniques 
using linearization of compressed video data against analyses of DICOM Raw Data with the manufacturer's CHIQ software package, thereby confirming the ability to provide reliable estimation of echo-power from DICOM JPEG images. Results also point to important acquisition conditions that must be respected in order to apply these more universal approaches for DCE-US analysis.

\section{Methods}

\section{Data acquisition and file formats}

Data were acquired with an US imaging system (Aplio 50, Toshiba, Tochigi, Japan) able to store DCE-US clips in DICOM Raw Data and DICOM JPEG formatted files. Throughout this article, dynamic range, DR, two-dimensional gain, 2DG, and frequency settings are cited without units, as displayed on the Aplio monitor. A linear probe (PLT-1202-S, Toshiba) was used with a transmit-frequency setting of h12.0 in Contrast Harmonic Imaging (CHI) mode. The gain was set at 92 and the mechanical index was fixed at a low level (MI=0.1) for each protocol to minimize bubble destruction. The time gain compensation (TGC) controls were placed in a centered and fixed position. Because the acquisition of DICOM JPEG and DICOM Raw Data sequences could not be started at exactly the same time with the Aplio 50 system, for each experiment, DICOM JPEG recording was initiated first and then DICOM Raw Data recording was activated.

The complex DICOM file format is one element of the DICOM standard that is widely used in clinical information management. In a DICOM formatted file, the DICOM File Meta Information (header) is followed by information coded as an ordered succession of DICOM Data Elements. Information can be freely accessed when it is in Data Elements, named Standard Data Elements, that are defined in the standard. Private Data Elements are defined by an implementer, such as a manufacturer, to communicate information that is not contained in Standard Data Elements. If the implementer does not publish information on the Private Data Elements, they cannot be freely accessed.

In the acquired DICOM JPEG files, images displayed by the US system were stored as a sequence of JPEG color images. The images contained annotations, a pseudocolored sub-image devoted to the display of the scanned area and a colorbar. Echographic data are usually quantized as 8-bit values prior to display. For the Toshiba Aplio 50, JPEG compression was lossy, with an image compression ratio of 20. The acquired (4-10 
images/s) and stored (18 images/s) frame rates were not equivalent, due to machine restrictions. Therefore, some images in each acquired sequence were duplicated multiple times in the corresponding stored sequence.

The DICOM Raw Data files that are available with the Aplio system do not use JPEG compression. DICOM Raw Data provides much better bit-depth resolution by storing 12-bit values. However, most of the information needed to read these Raw Data files is stored in Private Data Elements that can only be read with $\mathrm{CHI}-\mathrm{Q}$ software. In spite of this, it was possible to obtain sufficient image information on the DICOM Raw Data to perform image synchronization as described in the Data analysis section.

\section{Echo-power estimation approaches: PixPower, CHI-Q, VueBox ${ }^{\mathrm{TM}}$}

The link between a linear echo-power value, $P$, and the power in decibel $P_{d B}$ signal can be written as:

$$
P_{d B}=10 \log _{10} \frac{P}{P_{0}}
$$

where $P_{0}$ is the $0 \mathrm{~dB}$ reference power level. Linear power may be estimated from the power in decibels by using a straightforward inversion of Eq. (1):

$$
P=P_{0} 10^{\frac{P_{d B}}{10}}
$$

\section{PixPower, laboratory-based approach}

US systems typically provide logarithmically compressed video images in DICOM JPEG formatted files. Linearization is therefore required to reverse the log-compression and estimate the echo-power for each pixel of the ROI for each time point. In-house software, named PixPower, was developed to estimate power from DICOM JPEG acquisitions.

Images composed of JPEG pseudocolor data $I(x, t)$, where $x$ denotes the position of the pixel in the image and $t$ refers to the time point in the sequence, were transferred from the US system. First, to convert these RGB images into greyscale images, the standard-definition television luminance $I_{L}(x, t)$ was computed according to ITU-R Recommendation BT. 601-7 (2011). A greyscale lookup table was then extracted from the colorbar displayed on the original image and smoothed using the spline approximation $h:[0,1] \rightarrow\{0,255\}$. The inverse of this lookup table $h^{-1}$ was estimated. Finally, the local power for each pixel was calculated as follows 


$$
P_{P x P, d B}(x, t, \alpha, \beta)=\alpha \times D R \times\left(h^{-1}\left(I_{L}(x, t)\right)-1\right)+\beta
$$

where $D R$ is the displayed dynamic range. The two parameters $\alpha$ and $\beta$ are to be determined. The parameter $\alpha$ corrects the unitless value of dynamic range displayed onscreen (if $\alpha=1$, then the displayed $D R$ is equal to the real dynamic range in $\mathrm{dB}$ ), and $\beta$ is the relative scaling factor adjusting echo-power (expressed in arbitrary units) as calculated by different software to the same scale. As described in the Data analysis section, the parameters $\alpha_{P x P, d B}$ and $\beta_{P x P, d B}$ must be optimized relative to some reference measurements to enable correct quantification.

Over a selected ROI $\Omega$ of $|\Omega|$ pixels, the mean linear echo-power is then estimated by

$$
P_{P x P}(\Omega, t, \alpha, \beta)=\frac{1}{|\Omega|} \sum_{x \in \Omega} 10^{\frac{P_{P x P, d B}(x, t, \alpha, \beta)}{10}}
$$

Values of $P_{P x P}(\Omega, t)$ are then exported in a text file.

\section{CHI-Q (version 1.6)}

Toshiba proposes a quantification software called CHI-Q which only reads files recorded by Toshiba systems in DICOM Raw Data. For this work, version 1.6 of CHI-Q was used. ROIs cannot be saved or loaded in this software. For this study, a rectangular ROI of fixed dimensions was drawn manually by means of the $\mathrm{CHI}-\mathrm{Q}$ user-interface. An image from the sequence displaying the outline of the ROI was saved for subsequent automatic extraction of the ROI coordinates to define the $\Omega$, to be analyzed on the same data set by all of the software packages. The power estimated by $\mathrm{CHI}-\mathrm{Q}$ within the analysis region at each time $t$ throughout the sequence, $P_{C H I Q}(\Omega, t)$, was exported to a text file.

\section{VueBox $^{\mathrm{TM}}$ (version 4.2)}

VueBox $^{\mathrm{TM}}$ is a quantification software developed by Bracco Suisse SA that estimates echo-power values from video compressed images in DICOM JPEG files (Rognin et al.2010; Tranquart et al.2012). In this study, we used VueBox ${ }^{\mathrm{TM}}$ version 4.2. The software included calibration files for the US system, probe and dynamic ranges used in this study. These calibration files are necessary to translate pixel luminance values into linear echopower values. ROI coordinates were imported from the text file which contained the region coordinates manually selected for $\mathrm{CHI}-\mathrm{Q}$ analysis. Although available in VueBox ${ }^{\mathrm{TM}}$, motion compensation was not used in 
this study. The power estimated by VueBox ${ }^{\mathrm{TM}}$ within the analysis region at each instant $t$ of the sequence, $P_{V B}(\Omega, t)$, was exported to a text file.

\section{Dose ranging, an in vitro experiment}

A dose ranging experiment was conducted using the BR38 experimental US contrast agent (Bracco Suisse SA, Geneva, Switzerland) to assess the proportionality between microbubble concentration and echo-power estimated with the different approaches. BR38 microbubbles have an average diameter of $1.5 \mu \mathrm{m}$ and consist of a mixture of perfluorobutane and nitrogen, stabilized by a phospholipid shell (Schneider et al.2011). The agent was reconstituted prior to the experiment in $5 \mathrm{~mL}$ of physiological solution $(0.9 \% \mathrm{NaCl})$ to yield approximately $2 \times 10^{8}$ bubbles $/ \mathrm{mL}$. The microbubbles were then dispersed in an $800 \mathrm{~mL}$ beaker with deionised $0.9 \% \mathrm{NaCl}$ under constant magnetic stirring. The range of tested concentrations spanned dilutions between 1:400000 and 1:250 of the native reconstituted agent. These dilutions were obtained by successive addition of native reconstituted agent directly into the beaker using an appropriate micropipette.

The US probe was positioned onto an acoustically-transparent, $10 \mathrm{~mm}$-thick gelpad, such that its focal zone was immediately below the gelpad surface. The gelpad was used to separate the probe from the suspension in order to prevent attenuation of the US pulses by microbubbles in the path between the probe and the measurement region (focal zone). An attenuator was placed at the bottom of the $800 \mathrm{~mL}$ beaker to prevent reflection from this interface (Figure 2).

Data were acquired with an acquisition frequency of 10 frames/s at two different dynamic range settings: 60 and 80 . For each dilution and for each dynamic range, $N=40$ frames were recorded in DICOM Raw Data and DICOM JPEG formatted files. Data acquisition from all dilutions was performed within less than 15 minutes to ensure that concentrations were not depleted by microbubble-gas dissolution. Data were also recorded with the beaker filled only with the $0.9 \% \mathrm{NaCl}$ solution to provide a control measurement of the background echopower level (noise).

\section{The murine tumor model, an in vivo experiment}

As part of another study, contrast-enhanced sequences were acquired in both DICOM Raw Data and DICOM JPEG formatted files with a subcutaneous pancreatic adenocarcinoma model (MIA PaCa2 cells ectopically implanted in 13 nude mice, 15 days after cell injection). The animals were housed at the CEF (Centre 
d'Explorations Fonctionnelles of the Cordeliers' Research Center, Agreement no. A75-06-12). All experiments were conducted in accordance with the institutional guidelines and the recommendations for the care and use of laboratory animals established by the French Ministry of Agriculture (protocol authorization: p3/2009/010). For imaging, the dynamic range setting was fixed at 60 . The focal zone was set at the center of the tumor. The frame rate (4 frames/s) was set lower than for the previous in vitro data acquisition to minimize microbubble destruction in regions of slow capillary flow. Contrast US data sequences were acquired during the bolus passage of $100 \mu \mathrm{L}$ of SonoVue (Bracco Imaging SpA, Milan, Italy) followed by a destruction-reperfusion sequence. Twenty-four, 60-second sequences were used in this work.

\section{Data analysis}

For several reasons, temporally and spatially matched regions $\Omega$ of sequences from the same specimen stored in DICOM JPEG and DICOM Raw Data cannot be compared directly. As stated earlier, each pair of DICOM JPEG and DICOM Raw Data sequences overlap in time but begin and end at slightly different instants in time. The time intervals between two consecutive images stored in the two data files do not perfectly match either. Furthermore, the pixel sizes in the images displayed by $\mathrm{CHI}-\mathrm{Q}$ and those stored in the DICOM JPEG or DICOM Raw Data images are not the same. The fields of view are also slightly different. Therefore, before matched regions $\Omega$ could be selected, automatic image registration and automatic time synchronization of the sequences were necessary (details are provided in the Appendix). Once this had been achieved, ROIs delimited with $\mathrm{CHI}-\mathrm{Q}$ could be transferred onto the corresponding coordinates and times of the DICOM JPEG sequence. Reliable spatial matching and time-synchronization steps allowed a meaningful subsequent comparison of echo-power values obtained by the three quantification approaches because the values were obtained from matched regions and instants during contrast passage.

\section{The in vitro dose ranging experiment}

A rectangular ROI $1.2 \mathrm{~mm}$ deep by $13.2 \mathrm{~mm}$ wide was placed on images immediately below the interface between the gel-pad and the contrast solution. These dose-ranging data were initially used to determine scaling factors necessary to place the echo-power estimations provided by the different software packages on the same footing.

VueBox $^{\mathrm{TM}}$ analysis of a DICOM JPEG file provides power estimates within a ROI $\Omega$ at each instant $t_{n}$ in the sequence, $P_{V B}(\Omega, t)$. The estimated echo-power values are expressed in arbitrary units. In order to adjust the 
level of estimates obtained with the VueBox ${ }^{\mathrm{TM}}$ approach to the DICOM Raw Data based CHI-Q estimates (also in arbitrary units), a scaling factor was determined from the dose-ranging data acquired from all sequences with the same DR by:

$$
\beta_{V B}=\operatorname{argmin}_{\beta} \sum_{\Omega \in A} \frac{\frac{1}{N} \sum_{n=1}^{N}\left|\beta P_{V B}\left(\Omega, t_{n}\right)-P_{C H I Q}\left(\Omega, t_{n}\right)\right|}{\frac{1}{N} \sum_{n=1}^{N} P_{C H I Q}\left(\Omega, t_{n}\right)}
$$

where $A$ is the set of ROls $\Omega$. The denominator normalizes the mean power difference by the echo-power estimated by $\mathrm{CHI}-\mathrm{Q}$ to give stronger relative weight to differences at low power.

The in-laboratory approach, PixPower, based on DICOM JPEG analysis, requires not only optimization of a scaling factor, but also the correction of the unitless value $D R$ displayed onscreen by the imaging system to the true dynamic range in $\mathrm{dB}$. For each $\mathrm{DR}$, the best $(\alpha, \beta)$ parameters, $\left(\alpha_{P x P, d B}, \beta_{P x P, d B}\right)$, were determined by minimizing, for all the regions $\Omega$, the means of the relative errors between powers estimated by PixPower and CHI-Q:

$$
\left(\alpha_{P X P, d B}, \beta_{P X P, d B}\right)=\operatorname{argmin}_{(\alpha, \beta)} \sum_{\Omega \in A} \frac{\frac{1}{N} \sum_{n=1}^{N}\left|P_{P x P}\left(\Omega, t_{n}, \alpha, \beta\right)-P_{C H I Q}\left(\Omega, t_{n}\right)\right|}{\frac{1}{N} \sum_{n=1}^{N} P_{C H I Q}\left(\Omega, t_{n}\right)}
$$

The simplex method was used to perform this optimization.

For each concentration $c$, a ROI $\Omega_{c}$ was drawn and the average echo-power $P\left(\Omega_{c}, t\right)$ was calculated at each time point $t$ using the three different approaches. For each estimation approach, the resulting echo-power values from 40 -independent image frames was averaged to obtain the mean $\overline{P_{c}}$ and standard deviation $\sigma_{c}$. The average background noise level measured from ROls of 40-independent image frames in the saline-only solution at the same field position $\overline{P_{0}}$ and at the same dynamic range setting was subtracted from each $\bar{P}_{c}$ to correct the echo-power estimate for noise. The regression and error estimation were then performed using this corrected mean value, $\bar{P}_{c o r r, c}$ and standard deviation $\sigma_{c o r r, c}=\sqrt{\sigma\left(\Omega_{c}\right)^{2}+\sigma\left(\Omega_{0}\right)^{2}}$.

The $\bar{P}_{c o r r, c}$ values were then converted to dB. A linear fit was performed on the data to find the slope and the intercept describing the relationship between microbubble concentration $c$ and echo-power estimates for each approach. The corresponding coefficients of determination $\mathrm{R}^{2}$ were also calculated to assess how well the linear model fit each set of data. To estimate the error on these two regression parameters, the Monte Carlo method was applied. For each concentration, 40 random numbers $m_{c}$ were generated from the normal 
distribution with mean $\bar{P}_{c o r r, c}$ and standard deviation $\sigma_{c o r r, c}$. The average values $M_{c}$ were calculated and converted to $\mathrm{dB}$. A linear regression was performed using these $M_{C}$ values resulting in a slope and an intercept. The generation of random regression parameters based on the data distribution was repeated 10000 times. The distribution of these slopes and intercepts was estimated using a kernel density estimator. Finally, 95\% confidence intervals $(95 \% \mathrm{Cl})$ were calculated for the slope and the intercept.

\section{The in vivo murine tumor model experiment}

Using the synchronized DICOM Raw Data and DICOM JPEG sequences acquired in vivo, an independent comparison was made of echo-power values obtained with $\mathrm{CHI}-\mathrm{Q}$, VueBox ${ }^{\mathrm{TM}}$ and PixPower. Forty, independent ROIs (2-mm by $2-\mathrm{mm}$ ) were selected inside the tumors, in the coupling gel and in zones including strongly reflecting structures so that pixel levels were sampled with luminances covering the entire range from noise to saturation. Each region was transferred to all images throughout a DICOM Raw Data sequence and then transferred to time-matched and spatially matched positions of the corresponding DICOM JPEG sequence.

\section{Results}

\section{The dose-ranging experiment}

The correction parameters required to scale VueBox ${ }^{\mathrm{TM}}$ and PixPower echo-power estimations to $\mathrm{CHI}-\mathrm{Q}$ data are presented in Table 1. For VueBox ${ }^{\mathrm{TM}}$ data, the multiplicative factor $\beta_{V B}$ was calculated according to Equation 5. The optimal matching of VueBox ${ }^{\mathrm{TM}}$ estimates onto $\mathrm{CHI}-\mathrm{Q}$ values was obtained using $\beta_{V B}=1.2993 \times 10^{-6}$ and $\beta_{V B}=9.4440 \times 10^{-6}$ for the dynamic ranges DR60 and DR80, respectively. For the PixPower approaches, both $\alpha_{P x P, d B}$, the correction on the displayed DR, and $\beta_{P x P, d B}$ were calculated according to Equation 6 . The values for optimal adjustment of PixPower values onto $\mathrm{CHI}-\mathrm{Q}$ echo-power estimates were found to be $\left(\alpha_{P X P, d B}, \beta_{P X P, d B}\right)=(0.7740,-5.3358)$ and $\left(\alpha_{P X P, d B}, \beta_{P X P, d B}\right)=(0.7664,5.8604)$ for DR60 and DR80, respectively. Data linearization by PixPower was then performed using these values in Equation 3.

Echo-power estimates made from dose-ranging data using $\mathrm{CHI}-\mathrm{Q}$, VueBox ${ }^{\mathrm{TM}}$ and PixPower analysis are presented as a function of agent concentration at DR60 and DR80 (Figures 3 and 4, respectively). A linear regression was applied on the data after subtraction of background noise, at each DR, to assess linearity of the echo-power vs. concentration. The correlation of the results with the fit $y=b_{1}+b_{2} x$ was characterized with 
$\mathrm{R}^{2}$ values. Measurements were performed for $B R 38$ concentrations ranging from 500 microbubbles $/ \mathrm{mL}$ to $8 \times 10^{5}$ microbubbles $/ \mathrm{mL}$. The linear regression, at both DRs, for all three analysis approaches resulted in lines $\left(\mathrm{R}^{2} \geq 0.9996\right)$ with $95 \% \mathrm{Cl}$ for the slopes ranging from 9.22 to $9.68 \mathrm{~dB} /$ decade (Tables 2 and 3). Thus, $\mathrm{CHI}-\mathrm{Q}$, VueBox ${ }^{\mathrm{TM}}$ and DR-corrected PixPower allow relative concentration estimates within $0.8 \mathrm{~dB} /$ decade of the theoretically predicted power-concentration relationship (10 dB/decade, $p=0.05)$.

\section{The murine model experiment (VueBox ${ }^{\mathrm{TM}}$ and PixPower vs. CHI-Q)}

Data were acquired in vivo in a subcutaneous pancreatic adenocarcinoma model using a DR of 60. Echopower measurements were performed using forty independent ROIs from 24, 60-s long sequences presenting bolus passage of SonoVue followed by microbubble destruction and agent reperfusion.

Data from both VueBox ${ }^{\mathrm{TM}}$ and PixPower software packages were computed, as described in the previous section. Figure 5 shows an example of spatially-averaged echo-power kinetics obtained using $\mathrm{CHI}-\mathrm{Q}$ applied to DICOM Raw Data, and using VueBox ${ }^{\mathrm{TM}}$ and PixPower applied to DICOM JPEG data after spatial and temporal synchronization of an entire in vivo sequence. It shows the initial bolus arrival, followed by bubble destruction and replenishment within the $60 \mathrm{~s}$ interval examined.

The echo-power values obtained using VueBox ${ }^{\mathrm{TM}}$ and PixPower linearizing equations are compared to those obtained using $\mathrm{CHI}-\mathrm{Q}$ in Figure 6. The position of each point on the graph provides a comparison of the results obtained with CHI-Q analysis and with either VueBox ${ }^{\mathrm{TM}}(\mathrm{a}$ and $\mathrm{b}$ ) or PixPower (c and $d$ ) in corresponding ROIs at matched time $t$. A total of 9273 echo power estimates were extracted after synchronization from the 40 sequences of 60 seconds. Weak values of echo-power were obtained from regions placed in the coupling gel and from regions of tissue at time-points with low contrast concentration. High values were obtained during peak contrast passage and from regions placed to include highly reflective structures. Graphs $6 \mathrm{a}$ and $6 \mathrm{c}$ present linearized echo-power obtained with either VueBox ${ }^{\mathrm{TM}}$ or PixPower vs. $\mathrm{CHI}-\mathrm{Q}$ estimates, respectively. Graphs $6 \mathrm{~b}$ and $6 \mathrm{~d}$ are Bland-Altman plots showing the same measurements transformed in decibels. The difference between DICOM JPEG approaches and CHI-Q echo-power values is displayed against the corresponding mean echo-power value (Bland and Altman1999). Figures 6a and 6c show that both VueBox ${ }^{\mathrm{TM}}$ and PixPower echopower estimations are strongly and linearly related to the results obtained with CHI-Q $\left(R^{2}>0.999\right.$ with a slope of 0.96 for VueBox ${ }^{\mathrm{TM}}$ and 0.94 for PixPower). The color of the data points in graphs presented in Figure 6 are based on the probability distribution of the pixel luminances within each ROI. ROIs for data points displayed in 
blue present more than $98 \%$ pixels with a luminance $<30$ and ROls for data points in red more than $1 \%$ with a luminance $>254\left(I_{L}(x, t) \in[0,255]\right)$. Blue and red data points are thus affected by thresholding and saturation at the dynamic range limits, respectively. The Bland-Altman plots (Figures $6 \mathrm{~b}$ and $6 \mathrm{~d}$ ) show that these labeled data points diverge from the mean difference between the methods. Within the intermediate range (black data points) the mean difference $\left(P_{V B, d B}-P_{C H I Q, d B}\right)$ is $-0.25 \mathrm{~dB}$ with $95 \% \mathrm{Cl}$ from -0.75 to $0.26 \mathrm{~dB}$. Therefore, VueBox $^{\mathrm{TM}}$ echo-power estimates may be from $16 \%$ below to $6 \%$ above $\mathrm{CHI}-\mathrm{Q}$ results $(p=0.05)$. The mean difference $\left(P_{P x P, d B}-P_{C H I Q, d B}\right)$ was $-0.17 \mathrm{~dB}$ with $95 \% \mathrm{Cl}$ from -0.67 to $0.13 \mathrm{~dB}$. PixPower, therefore, can give estimates from $14 \%$ lower to $3 \%$ higher than $\mathrm{CHI}-\mathrm{Q}(\mathrm{p}=0.05)$.

\section{Discussion}

The objective of this study was to investigate if linearized, compressed US data can provide echo-power estimations as good as those provided by a manufacturer's software package applied to a Raw Data file. Simultaneously acquired compressed and Raw data obtained with the Toshiba Aplio 50 US system were used. Three approaches for estimating echo-power from contrast ultrasonographic images were compared using data acquired at the same time and in identical ROIs: 1) a dedicated CHI-Q software tool by Toshiba applied to DICOM Raw Data, 2) a commercially available VueBox ${ }^{\mathrm{TM}}$ software package by Bracco that applies algorithms to extract echo-power from DICOM JPEG images at the pixel-level and 3) an in-house software package (PixPower) that applies a linearizing equation to estimate the echo-power value for each pixel of DICOM JPEG images. This work validates that echo-power quantification from DCE-US can be applied, in our conditions, using compressed video images as well as with machine specific software and Raw Data files.

Results (Tables 2 and 3) from dose-ranging data confirmed that $\mathrm{CHI}-\mathrm{Q}$ is a good standard as it demonstrated strong linear relationships $\left(\mathrm{R}^{2}>0.999\right)$ between agent concentration and echo-power for both dynamic ranges tested (DR60 and DR80) with slopes within $0.8 \mathrm{~dB} /$ decade of the theoretically-predicted slope of $10 \mathrm{~dB} / \mathrm{decade}$ $(p=0.05)$. The quantification approaches for echo-power estimation from compressed video data (VueBox ${ }^{\mathrm{TM}}$ and PixPower) also provided clear linear relationships $\left(R^{2} \geq 0.9996\right)$ of the echo-power versus agent concentration for both DRs considered. The slopes of regression were within $0.7 \mathrm{~dB} /$ decade of the theoretical slope $(p=0.05)$.

VueBox $^{\mathrm{TM}}$ linearization was achieved using a calibration file specific to the US system, probe and dynamic range used for image acquisition to translate pixel luminance values into echo power levels. For the laboratory- 
made software, PixPower, the compression was hypothesized to be logarithmic and a linearizing equation was determined and applied, keeping the effective dynamic range $\alpha_{P x P, d B}$ as a floating parameter. The doseranging experiment was performed to find the best estimate of $\alpha_{P x P, d B}$ correcting the displayed dynamic range against the $\mathrm{CHI}-\mathrm{Q}$ gold-standard. The observed, effective dynamic range in $\mathrm{dB}$ was found to be equal to approximately $80 \%$ of the unitless value displayed on the system screen. Thus, a linearization based on parameters simply extracted using the displayed values does not correctly estimate echo-power. As theoretical and displayed parameters cannot be directly used for linearization, the determination of the correct linearizing parameters requires ground truth data to find an appropriate inversion of the log-compression. A posteriori, the hypothesis of a logarithmic compression was confirmed by the excellent agreement between PixPower analysis with both the manufacturer's raw data analysis and with experimental dose ranging.

The in vitro evaluation of the echo-power estimates obtained with each approach was performed for different concentrations of BR38 contrast microbubbles in suspension. For a contrast quantification approach in DCE-US to be valid, the echo-power measurement resulting from the contrast microbubbles' acoustic response must be predictably related to the agent concentration. This requirement is met under certain conditions. The ROI used to test this relationship for different data analysis approaches was defined to be a thin rectangular region providing a homogeneous signal and minimal effects from attenuation. Moderate stirring was applied to insure a homogeneous microbubble distribution without agent aggregation or solution turbulence. Dose ranging was performed over a range of concentrations as wide as possible. At the lowest concentrations considered, the signal approaches the noise floor and greater variability was observed due to the limited number of microbubbles in the resolution cell. At high concentrations, microbubbles may tend to aggregate even under stirring and shadowing may appear even in a thin ROI. Signals sufficiently above the noise and with minimal shadowing were obtained within a range of BR38 concentrations from 500 microbubbles $/ \mathrm{mL}$ to $8 \times 10^{5}$ microbubbles $/ \mathrm{mL}$, i.e. a 1:1600 range. This range includes the levels of concentrations typically encountered in DCE-US. BR38 was selected for this study because this phospholipidshelled agent offers sufficient stability in solution to perform this 15-minute long experiment (Schneider et al.2011). To further limit possible influence of any bubble degradation over time, the dilutions were made by successive addition of native reconstituted agent directly into the beaker and all measurements were performed within 15 minutes of the first addition of contrast agent. A frame rate of 10 frames/s was selected to limit both microbubble destruction and experiment duration. 
Even though the ROI used for dose-ranging analysis was deliberately selected rather shallow to minimize attenuation, low levels of acoustic shadowing occurred within the depth of the ROI at high microbubble concentrations. At low concentrations, noise slightly increased values. These two phenomena most likely contribute to the fact that the measured slopes of the echo-power as a function of microbubble concentration are lower than the theoretically predicted value of $10 \mathrm{~dB} /$ decade.

The in vivo experiment confirmed that it is possible to accurately estimate the linear power from DICOM JPEG images. In particular, echo-power monitored during contrast agent bolus passage followed by destruction and reperfusion in a tumor (DR60) showed excellent correspondence between the values estimated from DICOM JPEG data using VueBox ${ }^{\mathrm{TM}}$ and PixPower and those calculated with CHI-Q from Raw Data (Figure 5). Graphical comparison of 9273 estimates from 40 temporally and spatially matched ROls showed that both DICOM JPEG-based approaches demonstrated results strongly related to those of the $\mathrm{CHI}-\mathrm{Q}$ reference (Figure 6). Care was taken to include ROls such that the entire range of echo-powers was explored from noise in the coupling gel to saturation in the highly reflecting regions. The Bland-Altman plots (Figures $6 \mathrm{~b}$ and $6 \mathrm{~d}$ ) show that the measurements based on both DICOM JPEG approaches present average bias less than $1 \mathrm{~dB}$ with respect to $\mathrm{CHI}-\mathrm{Q}$ measurements. The absolute bias greatly increases at very high and very low echo-power values for which pixel luminance in the ROls saturated or thresholded, respectively. This observation confirms that thresholding and saturation are persistent problems in data linearization even when the processing steps are well accounted for, because information is irremediably lost. Acquisition of data with a sufficient dynamic range to record contrast intensity throughout a wide range of values should minimize the deleterious effect of thresholding and saturation on echo-power estimation. ROIs could also potentially be screened for pixels at the dynamic range limits so that such pixels would not be taken into account during data processing.

The importance of the dynamic range choice has been previously pointed out by Rognin et al. (2008) who demonstrated that quantification based on linearized video data can be equivalent to quantification based on raw RF data under certain dynamic range and gain restrictions. According to Rognin et al., the dynamic range of log-compression should be superior to $45 \mathrm{~dB}$ to limit thresholding of low pixel amplitudes and saturation of high pixel amplitudes. Second, the gain should be carefully set in such a way as to exploit the full dynamic range available, while avoiding saturation. The current study expands on the previous work, insofar as all instants of contrast uptake-kinetics are studied whereas, in Rognin et al.'s work, only perfusion parameters resulting from curve fitting were compared. 


\section{Conclusions and perspectives}

In this work, linearized data from compressed video images were shown to be able to provide echo-power estimates as good as dedicated software exploiting data with internal binary-representation allowing optimal inversion for echo-power assessment. Machine-specific software CHI-Q by Toshiba using DICOM Raw Data files was considered as the reference and was compared to two other approaches using DICOM JPEG files: the commercially-available VueBox ${ }^{\mathrm{TM}}$ package by Bracco and a laboratory-made software PixPower. In the latter method, linearizing parameters were determined with a dose-ranging experiment. Each approach was used to assess the proportionality between their echo-power estimates and contrast agent concentrations for two different values of dynamic range. These methods were then compared on in vivo data in tumors acquired after microbubble injection, including a destruction and reperfusion phase. All methods demonstrated proportionality with microbubble concentration in the dose-ranging experiment within $0.8 \mathrm{~dB} / \mathrm{decade}$ of the theoretical relationship. The in vivo quantification showed that, ignoring data near the limits of the dynamic range, echo-power estimates ranged from $16 \%$ below to $6 \%$ over $\mathrm{CHI}-\mathrm{Q}$ results with VueBox ${ }^{\mathrm{TM}}$ and $14 \%$ below to $3 \%$ over with PixPower.

This work shows that even though data may be somewhat deteriorated by the compression in DICOM JPEG files, it is possible to accurately estimate the linear power from compressed video images acquired with an appropriate DR value. In this work, the results indicate the conditions that need to be satisfied for linearization and comparison of echo-power data in DCE-US studies. First, the data must be acquired with a sufficient DR to minimize pixel luminance thresholding and saturation. Second, automatic image registration techniques are required to faithfully compare data analyzed with different software. Calibration (VueBox ${ }^{\mathrm{TM}}$ ) or ground truth data (PixPower) is required to appropriately linearize compressed data. Finally, if several techniques are used for linearization within the same study, scaling factors between the values expressed by different software must be determined.

Wider access to reliable information from US system manufacturers about the compression of echo-power data that clinicians acquire would contribute substantially to facilitate correct estimation of echo-power. Manufacturers compliance to a standardized, high-precision "Raw Data" format suitable for DCE-US quantification requirements would also significantly empower multicentric studies. 


\section{Acknowledgements}

The BR38 microbubbles and VueBox ${ }^{\mathrm{TM}}$ software were kindly provided by Bracco Suisse SA. The authors are most grateful to Laurent Mercier of Bracco Suisse SA for advice on the use of VueBox. They also gratefully acknowledge the excellent technical assistance of the staff of the Centre d'Exploration Fonctionnelle, Cordelier's Research Center, for support with animal care. The authors acknowledge financial support from the Martine Midy Foundation, in particular for the fellowship of M. Lamuraglia. 


\section{Appendix :}

\section{Data synchronization}

As described in the Methods, the present comparison of quantification approaches was rather tricky, due to differences in spatial and temporal sampling of the sequences displayed by CHI-Q, stored in DICOM JPEG and stored in DICOM Raw Data. These sequences do not match spatially (pixel size and coordinates) or temporally (sequences beginning and end, intervals between consecutive images). Therefore, reliable and automatic image registration and time synchronization had to be applied for meaningful comparison, throughout all DICOM Raw Data and DICOM JPEG sequences, both on the dose ranging and the in vivo datasets.

Once image registration and time synchronization of the sequences had been achieved, ROls delimited with $\mathrm{CHI}-\mathrm{Q}$ could be transferred onto the corresponding coordinates and times of the DICOM JPEG sequence.

Image registration

Image registration is the process of aligning different images of the same scene using geometrical transformations. In this work, image registration was performed to locate matched points on corresponding images as displayed by CHI-Q, as stored in DICOM Raw Data files and, finally as stored in DICOM JPEG files. Sufficient information on the DICOM Raw Data coordinate mapping was thus obtained to allow image comparison with JPEG images and $\mathrm{CHI}-\mathrm{Q}$ display based on mutual information. To ensure that registration was performed at matched acquisition instants, only the first reperfusion frames acquired in each murine destruction-reperfusion sequence were used to find the correct transformations. These frames are easily identifiable on the DICOM JPEG and DICOM Raw Data sequences, due to the high-MI agent destruction either marked by a flash (DICOM JPEG) or by a larger time delay between consecutive frames (DICOM Raw Data and CHI-Q displayed images) that immediately precedes replenishment. Using elastix software (Klein et al.2010) and mutual information registration criteria, the best affine spatial transform was estimated to best match the DICOM JPEG image onto the CHI-Q displayed image. The final transform $T_{C \rightarrow J}$ was the mean of the affine transformations estimated from the first reperfusion images of the 24 different sets of acquired data. The same method was used to find the best affine spatial transform $T_{R \rightarrow J}$ to match the DICOM JPEG coordinates onto the DICOM Raw Data coordinates.

\section{Sequence synchronization}

$T_{R \rightarrow J}$ was applied to every image stored in DICOM JPEG to map to the same coordinate system as the DICOM Raw Data images. Redundant DICOM JPEG images were suppressed and the overlapping portions of each pair of sequences were identified. Sequence synchronization was performed on aligned sequences based on mutual information. In the work reported here, only these synchronous parts of each data set are considered.

\section{Transfer of ROIs}

Once the synchronous image pairs were identified, rectangular ROls were delimited using $\mathrm{CHI}-\mathrm{Q}$ and the image displayed by $\mathrm{CHI}-\mathrm{Q}$ was stored. The $\mathrm{CHI}-\mathrm{Q}$ image was then analyzed and the coordinates of the 
rectangular region's vertices were automatically identified. The coordinates of the position of the vertices in the DICOM JPEG image were computed using the transform $T_{C \rightarrow J}$ and stored in two text files. The first was formatted to be read by PixPower and the other by VueBox ${ }^{\mathrm{TM}}$. After the ROI transfer, echo-power estimates could be obtained within regions $\Omega$ of the JPEG sequences that were synchronized and spatially-matched to the data analyzed by CHI-Q software on DICOM Raw Data files. 


\section{REFERENCES}

ITU-R Rec.BT. 601-7 "Studio Encoding Parameters of Digital Television for Standard 4: 3 And Widescreen 16: 9 Aspect Ratios". 2011;

Arditi M, Frinking PJ, Zhou X, Rognin NG. A new formalism for the quantification of tissue perfusion by the destruction-replenishment method in contrast ultrasound imaging. IEEE Trans.Ultrason.Ferroelectr.Freq.Control 2006;53:1118-29.

Bland JM, Altman DG. Measuring agreement in method comparison studies. Stat.Methods Med.Res. 1999;8:135-60.

Blankstein R, Shturman LD, Rogers IS, Rocha-Filho JA, Okada DR, Sarwar A, Soni AV, Bezerra H, Ghoshhajra BB, Petranovic M, Loureiro R, Feuchtner G, Gewirtz H, Hoffmann U, Mamuya WS, Brady TJ, Cury RC. Adenosine-induced stress myocardial perfusion imaging using dual-source cardiac computed tomography. J.Am.Coll.Cardiol. 2009;54:1072-84.

Chung AS, Lee J, Ferrara N. Targeting the tumour vasculature: insights from physiological angiogenesis. Nat.Rev.Cancer 2010;10:505-14.

de Jong N, Hoff L. Ultrasound scattering properties of Albunex microspheres. Ultrasonics 1993;31:175-81.

Dietrich CF, Averkiou MA, Correas JM, Lassau N, Leen E, Piscaglia F. An EFSUMB introduction into Dynamic Contrast-Enhanced Ultrasound (DCE-US) for quantification of tumour perfusion. Ultraschall Med. 2012;33:344-51.

Folkman J. Angiogenesis in cancer, vascular, rheumatoid and other disease. Nat.Med. 1995;1:27-31.

Gauthier M, Tabarout F, Leguerney I, Polrot M, Pitre S, Peronneau P, Lassau N. Assessment of quantitative perfusion parameters by dynamic contrast-enhanced sonography using a deconvolution method: an in vitro and in vivo study. J.Ultrasound Med. 2012a;31:595-608.

Gauthier TP, Averkiou MA, Leen EL. Perfusion quantification using dynamic contrast-enhanced ultrasound: the impact of dynamic range and gain on time-intensity curves. Ultrasonics 2011;51:1026.

Gauthier TP, Chebil M, Peronneau P, Lassau N. In vitro evaluation of the impact of ultrasound scanner settings and contrast bolus volume on time-intensity curves. Ultrasonics 2012b;52:12-9.

Guibal A, Taillade L, Mule S, Comperat E, Badachi Y, Golmard JL, Le Guillou-Buffello D, Rixe O, Bridal $S L$, Lucidarme $O$. Noninvasive contrast-enhanced US quantitative assessment of tumor microcirculation in a murine model: effect of discontinuing anti-VEGF therapy. Radiology 2010;254:420-9.

Hudson JM, Williams R, Lloyd B, Atri M, Kim TK, Bjarnason G, Burns PN. Improved flow measurement using microbubble contrast agents and disruption-replenishment: clinical application to tumour monitoring. Ultrasound Med.Biol. 2011;37:1210-21.

Jain RK, Schlenger K, Hockel M, Yuan F. Quantitative angiogenesis assays: progress and problems. Nat.Med. 1997;3:1203-8. 
Klein S, Staring M, Murphy K, Viergever MA, Pluim JP. elastix: a toolbox for intensity-based medical image registration. IEEE Trans.Med.Imaging 2010;29:196-205.

Kremkau F. Diagnostic ultrasound: principles and instruments. Philadelphia: W.B. Saunders, 1998.

Krix M, Kiessling F, Farhan N, Schmidt K, Hoffend J, Delorme S. A multivessel model describing replenishment kinetics of ultrasound contrast agent for quantification of tissue perfusion. Ultrasound Med Biol 2003;29:1421-30.

Lampaskis M, Averkiou M. Investigation of the relationship of nonlinear backscattered ultrasound intensity with microbubble concentration at low MI. Ultrasound Med.Biol. 2010;36:306-12.

Lamuraglia M, Bridal SL, Santin M, Izzi G, Rixe O, Paradiso A, Lucidarme O. Clinical relevance of contrast-enhanced ultrasound in monitoring anti-angiogenic therapy of cancer: Current status and perspectives. Crit Rev.Oncol.Hematol. 2010;73:202-12.

Lassau N, Chami L, Benatsou B, Peronneau P, Roche A. Dynamic contrast-enhanced ultrasonography (DCE-US) with quantification of tumor perfusion: a new diagnostic tool to evaluate the early effects of antiangiogenic treatment. Eur.Radiol. 2007;17 Suppl 6:F89-F98.

Leen E, Averkiou M, Arditi M, Burns P, Bokor D, Gauthier T, Kono Y, Lucidarme O. Dynamic contrast enhanced ultrasound assessment of the vascular effects of novel therapeutics in early stage trials. Eur.Radiol. 2012;22:1442-50.

Lucidarme O, Correas JM, Bridal SL, Berger G. Quantification of ultrasound contrast agent response: comparison of continuous wave Doppler and power Doppler to backscattered radiofrequency data. Ultrasound Med.Biol. 2001;27:1379-86.

Lucidarme O, Franchi-Abella S, Correas JM, Bridal SL, Kurtisovski E, Berger G. Blood flow quantification with contrast-enhanced US: "entrance in the section" phenomenon--phantom and rabbit study. Radiology 2003;228:473-9.

Moran CM, Watson RJ, Fox KA, McDicken WN. In vitro acoustic characterisation of four intravenous ultrasonic contrast agents at $30 \mathrm{MHz}$. Ultrasound Med.Biol. 2002;28:785-91.

Peronneau P, Lassau N, Leguerney I, Roche A, Cosgrove D. Contrast ultrasonography: necessity of linear data processing for the quantification of tumor vascularization. Ultraschall Med. 2010;31:3708.

Powers JE, Phillips DJ, Brandestini MA, Sigelmann RA. Ultrasound Phased Array Delay Lines Based on Quadrature Sampling Techniques. Sonics and Ultrasonics, IEEE Transactions on 1980;27:287-94.

Quaia E. Microbubble ultrasound contrast agents: an update. Eur.Radiol. 2007;17:1995-2008.

Rognin NG, Arditi M, Mercier L, Frinking PJ, Schneider M, Perrenoud G, Anaye A, Meuwly JY, Tranquart F. Parametric imaging for characterizing focal liver lesions in contrast-enhanced ultrasound. IEEE Trans.Ultrason.Ferroelectr.Freq.Control 2010;57:2503-11.

Rognin NG, Frinking P, Costa M, Arditi M. In-vivo Perfusion Quantification by Contrast Ultrasound: Validation of the Use of Linearized Video Data Vs. Raw RF Data. IEEE Ultrasonics Symposium 2008;November:1690-3. 
Sanches JM, Marques JS. Compensation of log-compressed images for 3-D ultrasound. Ultrasound Med.Biol. 2003;29:239-53.

Schneider M, Anantharam B, Arditi M, Bokor D, Broillet A, Bussat P, Fouillet X, Frinking P, Tardy I, Terrettaz J, Senior R, Tranquart F. BR38, a new ultrasound blood pool agent. Invest Radiol. 2011;46:486-94.

Tranquart F, Mercier L, Frinking P, Gaud E, Arditi M. Perfusion quantification in contrast-enhanced ultrasound (CEUS)--ready for research projects and routine clinical use. Ultraschall Med. 2012;33 Suppl 1:S31-S38.

Wei K, Jayaweera AR, Firoozan S, Linka A, Skyba DM, Kaul S. Quantification of myocardial blood flow with ultrasound-induced destruction of microbubbles administered as a constant venous infusion. Circulation 1998;97:473-83. 


\section{Tables:}

Table 1: Adjustment parameters for dynamic ranges, DR60 and DR80. The adjustment parameters $\beta_{\mathrm{VB}}$ and $\beta_{\mathrm{PXP}, \mathrm{dB}}$ are the linear scaling factors optimized to adjust the level of echo-power estimates in arbitrary units from VueBox ${ }^{\mathrm{TM}}$ and PixPower to the level of the echo-power in arbitrary units estimated by $\mathrm{CHI}-\mathrm{Q}$. The DR correction parameter $\alpha_{\mathrm{PXP}, \mathrm{dB}}$ was empirically determined for each DR setting from dose-ranging data.

\begin{tabular}{|c|c|c|c|}
\cline { 3 - 4 } \multicolumn{2}{c|}{} & DR60 & DR80 \\
\hline VueBox & $\beta_{V B}$ & $1.2993 \cdot 10^{-6}$ & $9.4440 \cdot 10^{-6}$ \\
\hline \multirow{2}{*}{ PixPower } & $\alpha_{P x P, d B}$ & 0.7740 & 0.7664 \\
\cline { 2 - 4 } & $\beta_{P x P, d B}$ & -5.3358 & 5.8604 \\
\hline
\end{tabular}

Table 2: Linear regression parameters calculated for the echo-power as a function of contrast agent dose with each of the three approaches (DR60). The coefficient of determination $R^{2}$ is presented. The regression coefficients $b_{1}$ and $b_{2}$ used in the $y=b_{1}+b_{2} x$ model are shown along with the corresponding $95 \%$ confidence intervals $(95 \% \mathrm{Cl})$ calculated with the Monte Carlo method.

\begin{tabular}{|c|c|c|c|c|c|}
\hline \multirow[t]{2}{*}{ DR60 } & \multirow[t]{2}{*}{$R^{2}$} & \multicolumn{2}{|c|}{$b_{1}(d B)$} & \multicolumn{2}{|c|}{$\mathrm{b}_{2}$ (dB×mL/microbubbles) } \\
\hline & & Fit value & $95 \% \mathrm{Cl}$ & Fit value & $95 \% \mathrm{Cl}$ \\
\hline $\mathrm{CHI}-\mathrm{Q}$ & 0.9996 & -77.48 & $\begin{array}{c}{[-78.11 ;-} \\
76.76]\end{array}$ & 9.37 & {$[9.22 ; 9.50]$} \\
\hline VueBox $^{\mathrm{TM}}$ & 0.9996 & -77.54 & $\begin{array}{c}{[-78.28 ;-} \\
76.97]\end{array}$ & 9.41 & {$[9.30 ; 9.56]$} \\
\hline PixPower & 0.9996 & -78.15 & $\begin{array}{c}{[-78.80 ;-} \\
77.40]\end{array}$ & 9.50 & {$[9.35 ; 9.63]$} \\
\hline
\end{tabular}

Table 3: Linear regression parameters calculated for the echo-power as a function of contrast agent dose with each of the three approaches (DR80). The coefficient of determination $R^{2}$ is presented. The regression coefficients $b_{1}$ and $b_{2}$ used in the $y=b_{1}+b_{2} x$ model are shown along with the corresponding $95 \%$ confidence intervals $(95 \% \mathrm{Cl})$ calculated with the Monte Carlo method.

\begin{tabular}{|c|c|c|c|c|c|}
\hline \multirow[t]{2}{*}{ DR80 } & \multirow[t]{2}{*}{$R^{2}$} & \multicolumn{2}{|c|}{$b_{1}(d B)$} & \multicolumn{2}{|c|}{$\mathrm{b}_{2}$ (dB×mL/microbubbles) } \\
\hline & & Fit value & $95 \% \mathrm{Cl}$ & Fit value & $95 \% \mathrm{Cl}$ \\
\hline CHI-Q & 0.9998 & -77.99 & $\begin{array}{c}{[-78.54 ;-} \\
77.23]\end{array}$ & 9.46 & {$[9.29 ; 9.57]$} \\
\hline VueBox $^{\mathrm{TM}}$ & 0.9998 & -78.11 & $\begin{array}{c}{[-78.72 ;-} \\
77.47]\end{array}$ & 9.50 & {$[9.37 ; 9.62]$} \\
\hline PixPower & 0.9996 & -78.29 & $\begin{array}{c}{[-79.07 ;-} \\
77.68]\end{array}$ & 9.51 & {$[9.38 ; 9.68]$} \\
\hline
\end{tabular}




\section{Figure captions:}

Figure 1: Block diagram relating the main processing system steps after echo reception to image display and file storage for Raw Data and JPEG file formats. The software applied in this work to linearize each type of file is shown with a note on its limitations: machine-specific, requires calibration files for the machine and settings used, requires empirical calibration relative to dose-ranging experiments.

Figure 2: Diagram presenting the setup of the dose-ranging experiment in which the echo-power of solutions of different concentrations of BR38 contrast agent were measured for two dynamic ranges (60 and 80).

Figure 3: For data acquired at DR60, echo-power estimates after noise subtraction as a function of the number of BR38 microbubbles per $\mathrm{mL}$ of solution. Results obtained at each concentration are presented for the three echo-power estimation approaches: $\mathrm{CHI}-\mathrm{Q}$ (blue), VueBox ${ }^{\mathrm{TM}}$ (green) and PixPower (red). The linear fits are also shown.

Figure 4: For data acquired at DR80, echo-power estimates after noise subtraction as a function of the number of BR38 microbubbles per $\mathrm{mL}$ of solution. Results obtained at each concentration are presented for the three echo-power estimation approaches: $\mathrm{CHI}-\mathrm{Q}$ (blue), VueBox ${ }^{\mathrm{TM}}$ (green) and PixPower (red). The linear fits are also shown.

Figure 5: Example of an echo-power versus time curves from synchronized in vivo sequences measured using CHI-Q (blue), VueBox ${ }^{\mathrm{TM}}$ (green) and PixPower (red). The curves are well synchronized during the bolus passage as well as for the bubble destruction and the replenishment phases.

Figure 6: Comparison between echo-power estimates calculated on in vivo data with CHI-Q using DICOM Raw Data and DICOM JPEG-based methods: VueBox ${ }^{\mathrm{TM}}$ ( $a$ and b) and PixPower (c and d). On the left, the echopower estimates are displayed on a linear scale with the identity line in red (a and $c)$. On the right, the corresponding Bland-Altman plots show the difference of the power expressed in $\mathrm{dB}$ as a function of the mean of the power expressed in dB obtained with $\mathrm{CHI}-\mathrm{Q}$ and VueBox (b) or CHI-Q and PixPower (d). ROls for data points displayed in blue present more than $98 \%$ pixels with a luminance $<30$ and ROIs for data points in red more than $1 \%$ with a luminance $>254$. 
Figure 1:

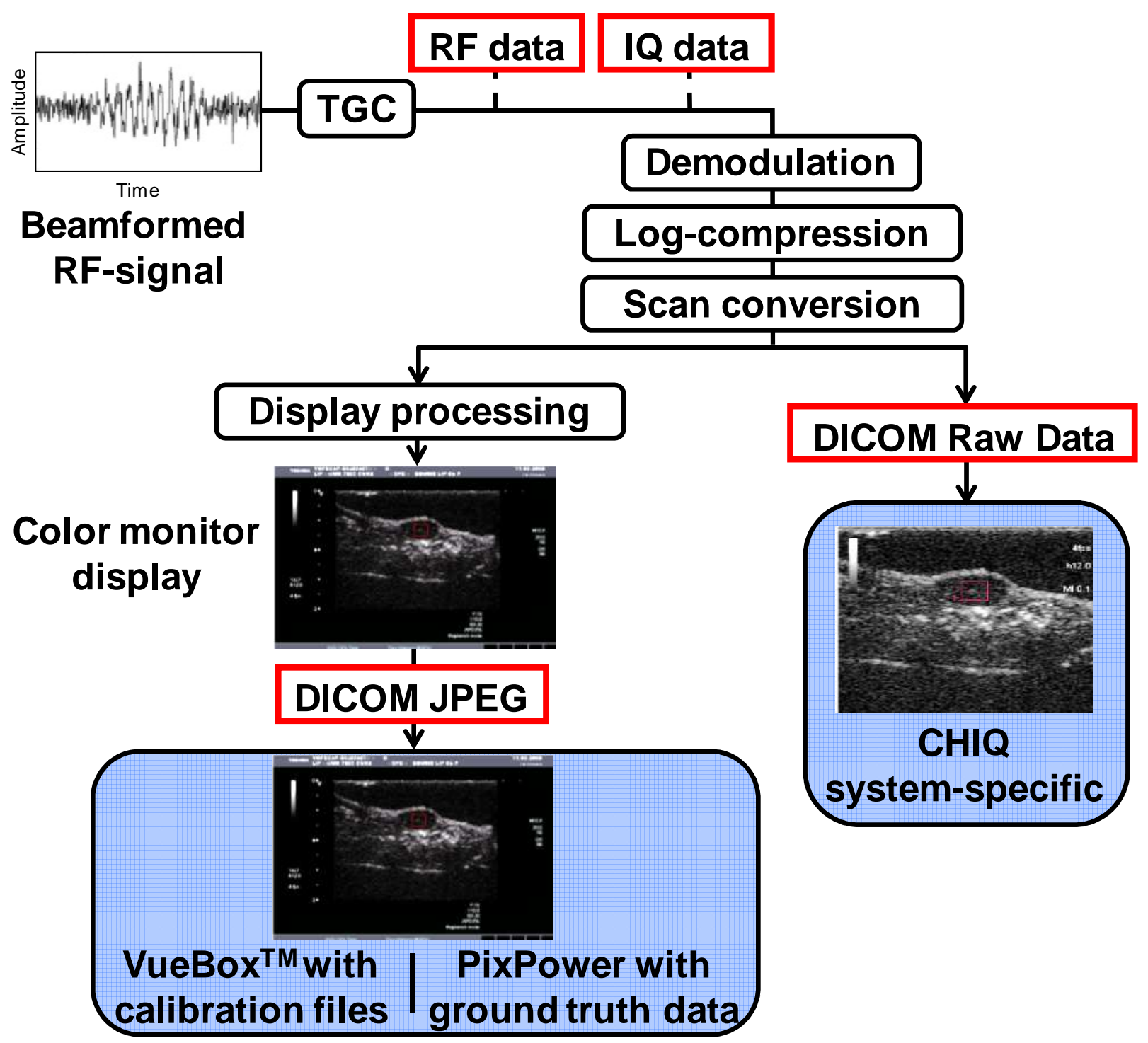


Figure 2:

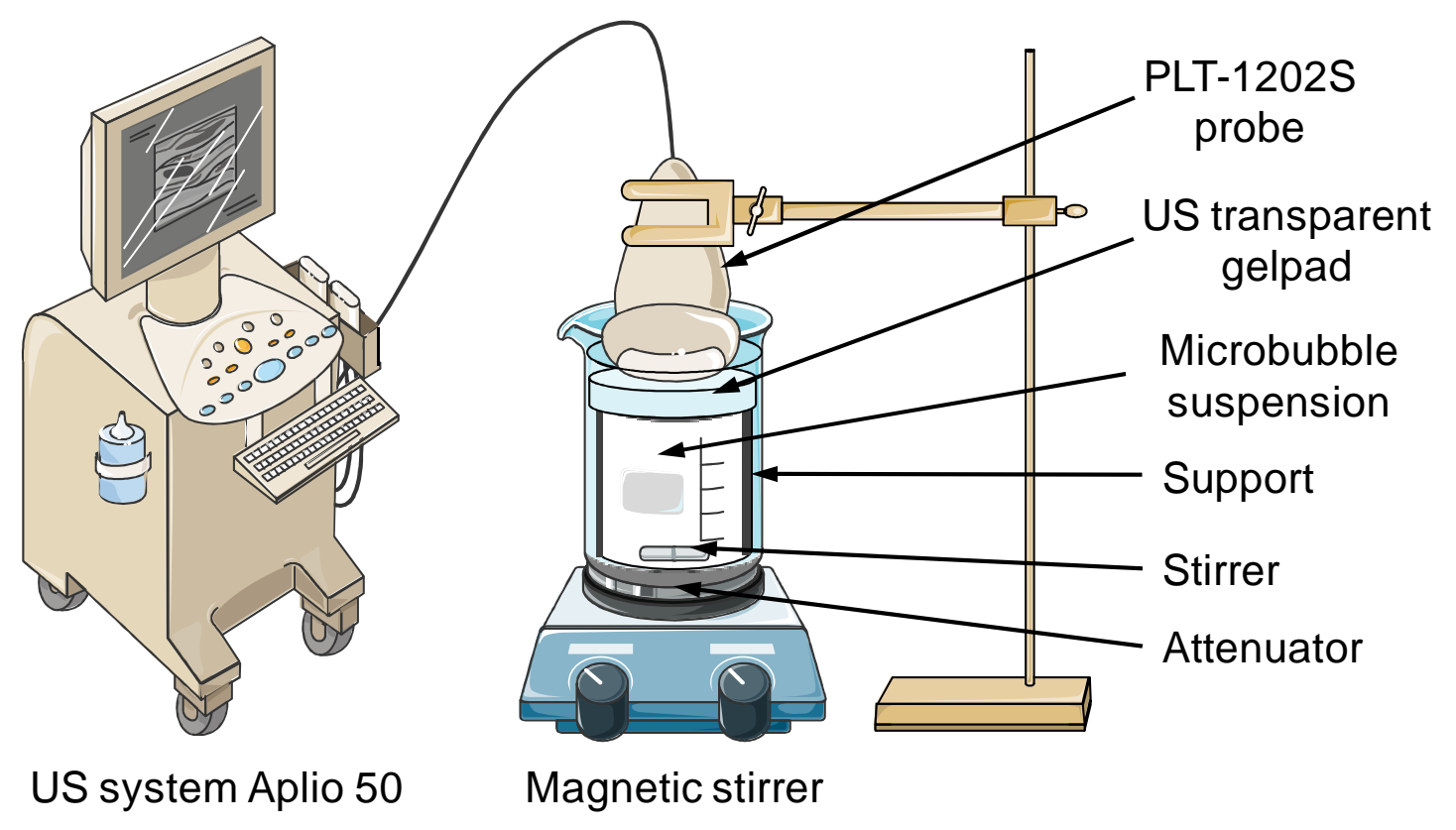


Figure 3:

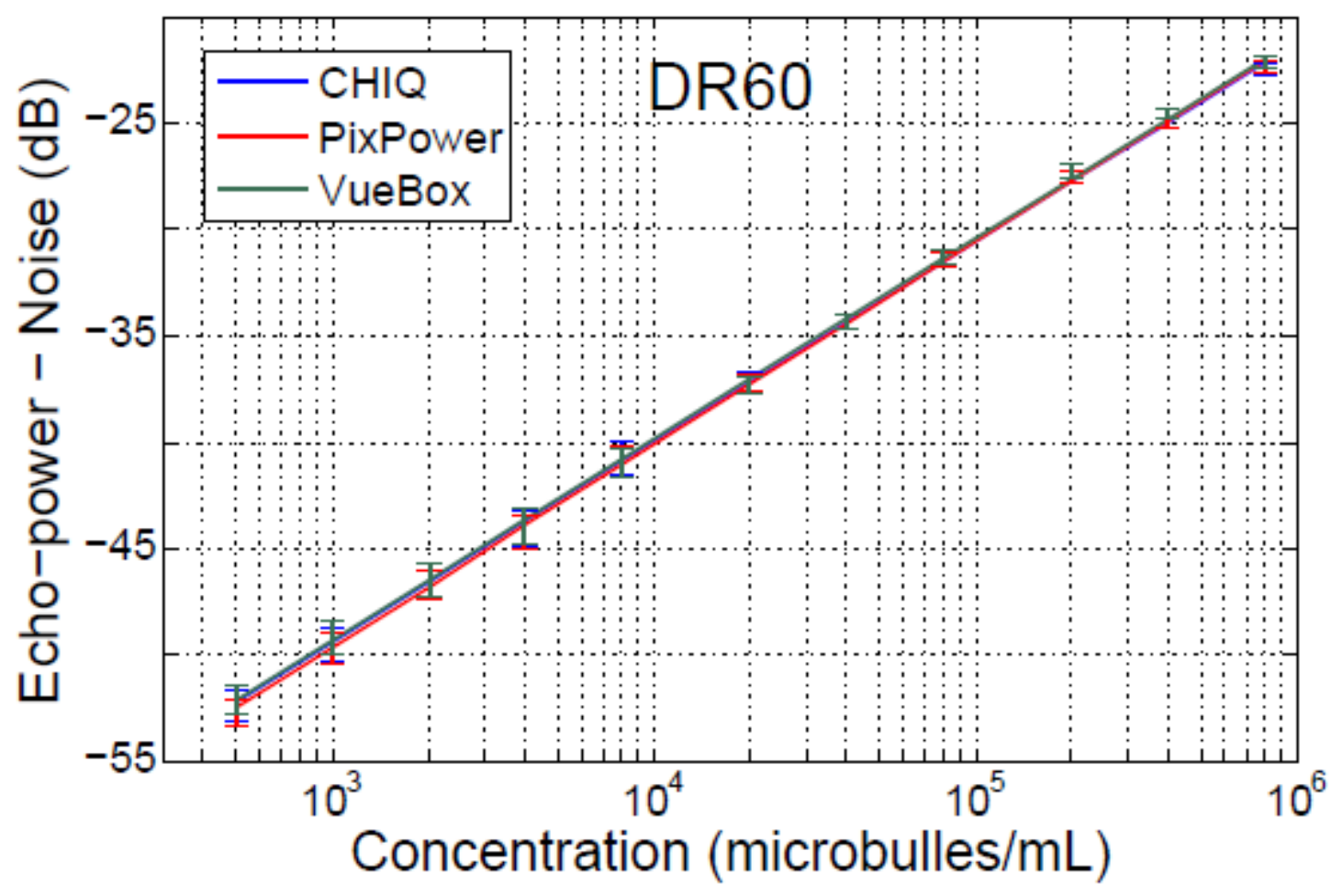

Figure 4:

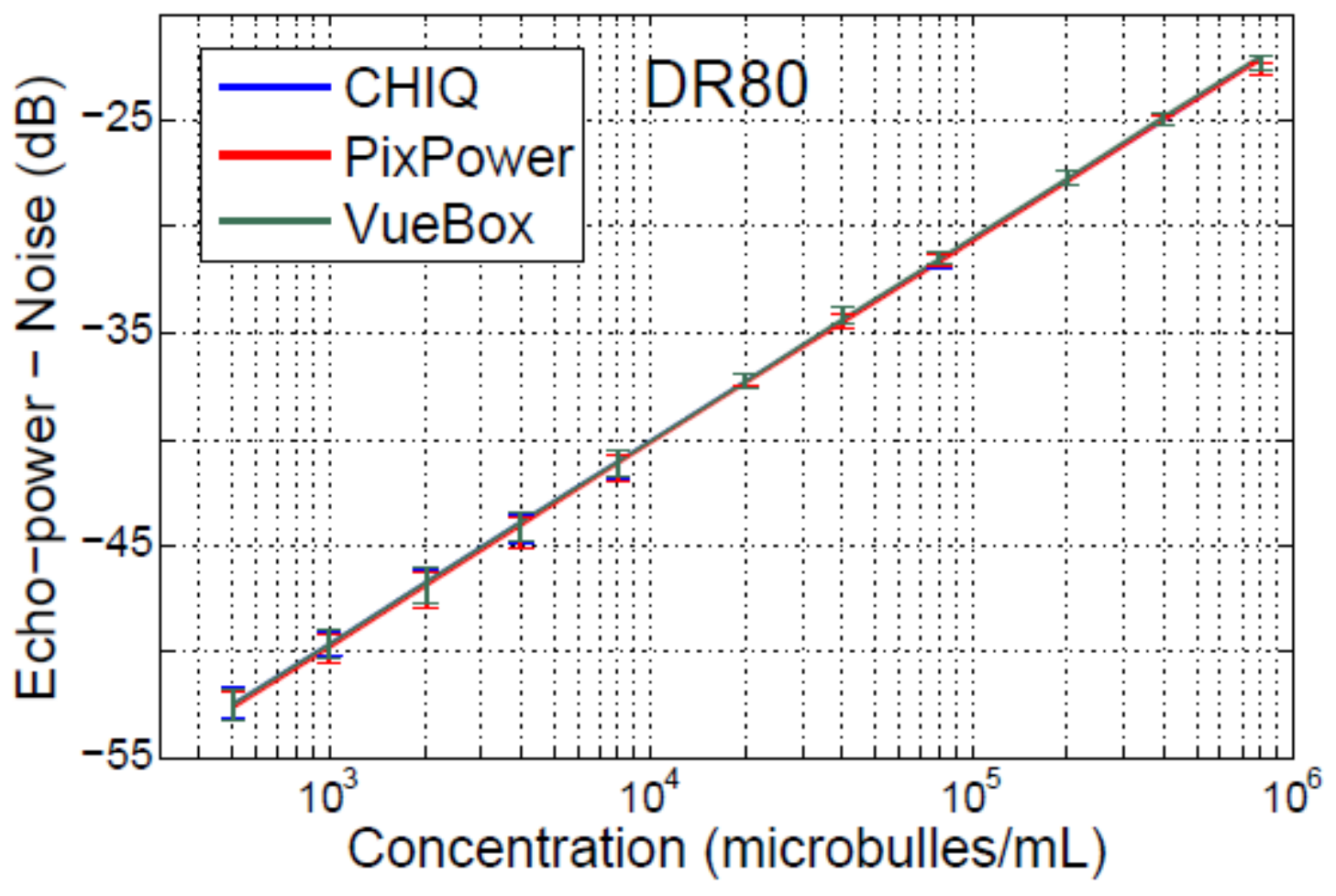


Figure 5:

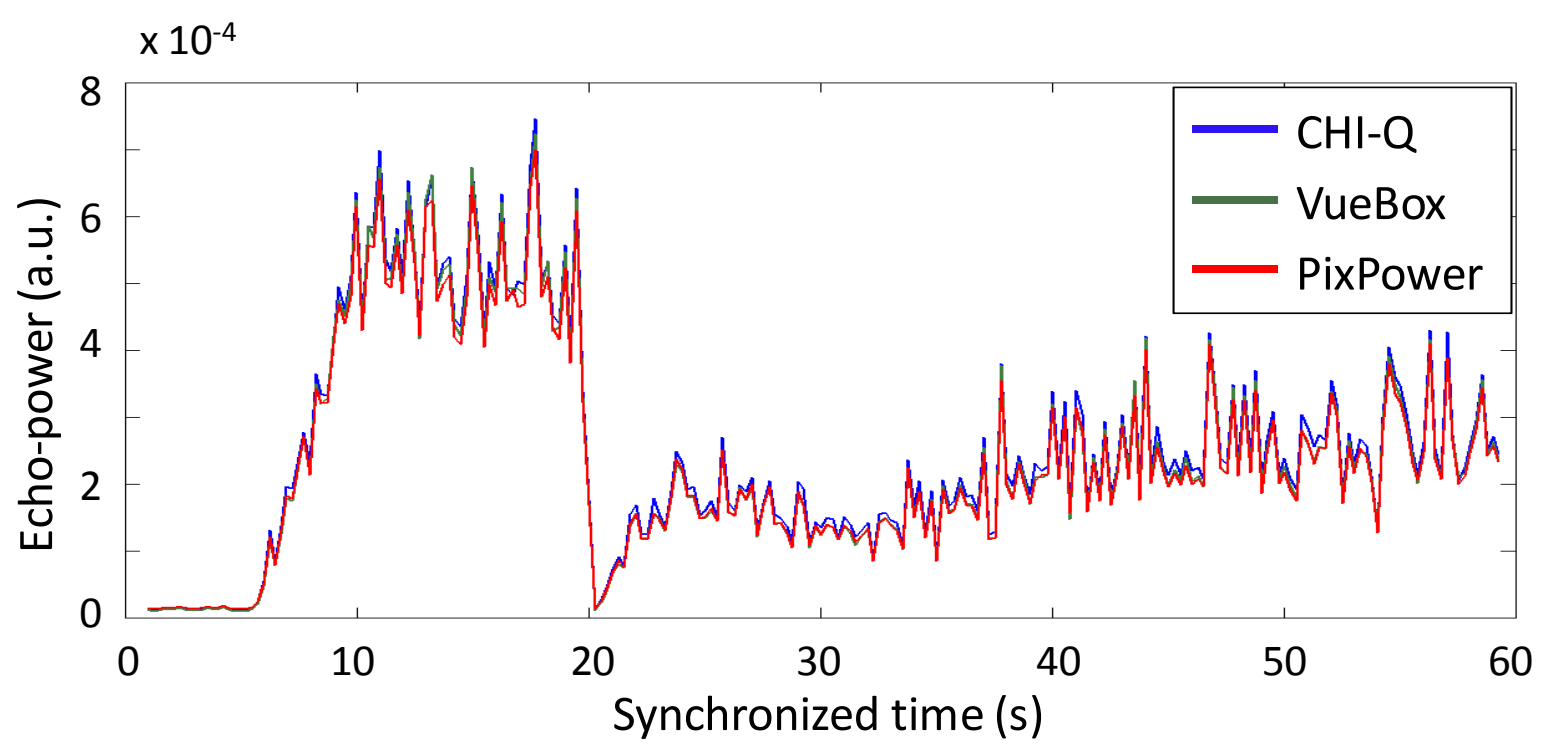


Figure 6:

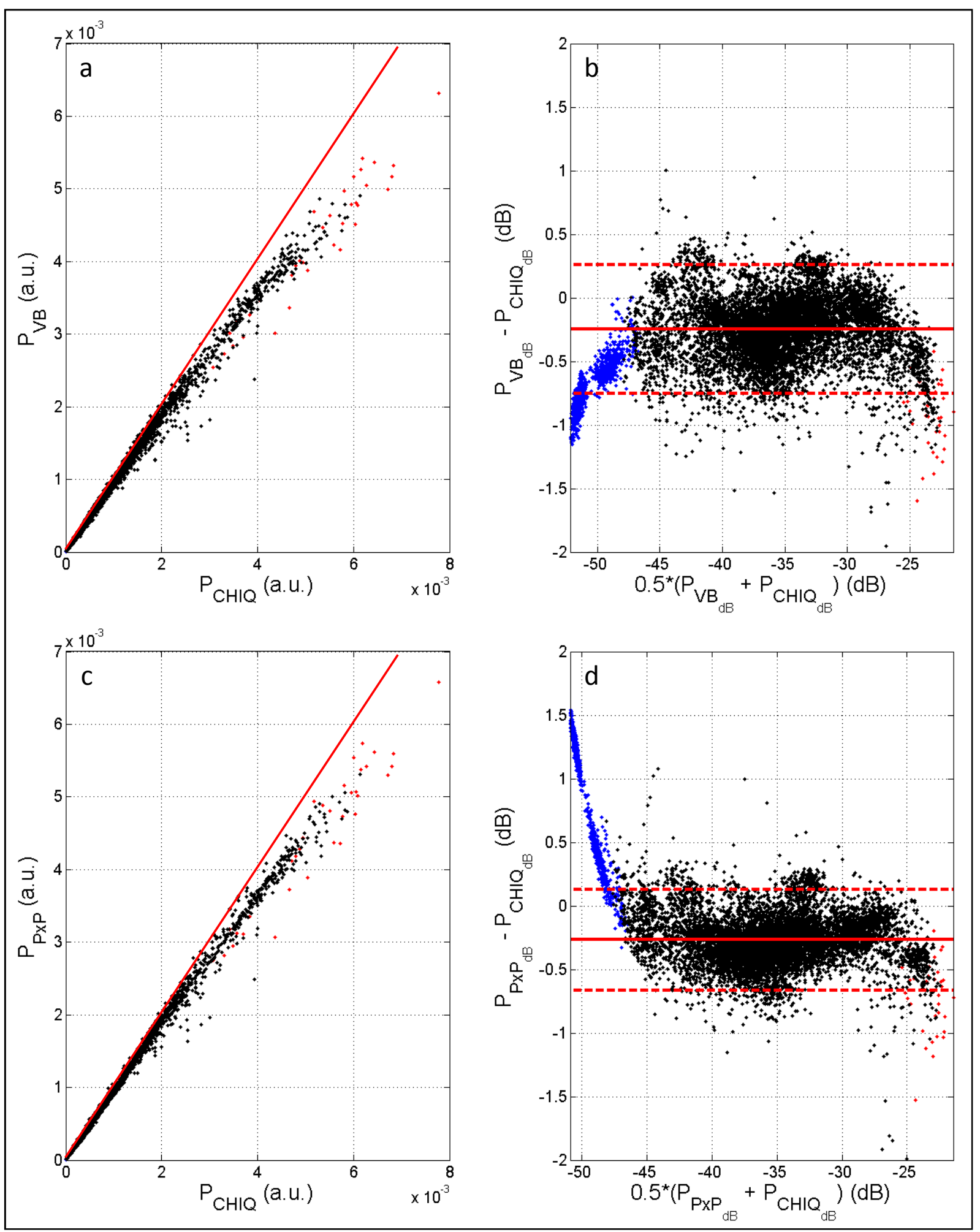

29 